\title{
Relaciones numéricas: matemática en los dígitos del año 2020
}

Numerical relations: mathematics in the digits of the year 2020

\section{Lorenzo Caballero Vigil $^{1}$, Reyna I. Jaramillo H. ${ }^{2}$ y Dalys E. Villarreal B. ${ }^{3}$}

1. Universidad de Panamá. Extensión Universitaria de Soná, Veraguas. Facultad de Ciencias Naturales, Exactas y Tecnología. Escuela de Matemática. lcaballero8015@hotmail.com https://orcid.org/0000-0003-0758-7038

2. Universidad de Panamá. Centro Regional Universitario de Azuero. Facultad de Ciencias Naturales, Exactas y Tecnología. Escuela de Matemática. reynaijaramillo@hotmail.com https://orcid.org/0000-0002-4096-5830

3. Universidad de Panamá. Centro Regional Universitario de Azuero. Facultad de Ciencias Naturales, Exactas y Tecnología. Escuela de Matemática. dalysvb23@outlook.com https://orcid.org/0000-0002-8132-1900

\section{Págs.: 86 - 96}

Recibido: $15 / 8 / 2020$

Aprobado: 28/8/2020

\section{Resumen}

Es por todos conocidos que la asignatura de matemática no goza del agrado ni favoritismo de la población estudiantil ni de la población en general, independientemente del nivel educativo donde estemos ubicados; por tanto, el docente de matemática debe apoyarse en diversas estrategias didácticas que le permitan captar la atención del estudiantado para traerlo de vuelta al aula de clases. Es por ello, que en este artículo presentamos una situación matemática potencialmente significativa, basándonos en el estudio de las relaciones numéricas que se presentan en las fechas del calendario, específicamente las que se dan en los dígitos del año 2020, como un apoyo para que cuando el docente de matemática imparta su clase pueda introducir conceptos o reforzar los ya aprendidos de una forma dinámica y motivadora.

Palabras clave: relaciones numéricas, situaciones matemáticas, motivación matemática. 


\begin{abstract}
It is by all known that the subject of mathematics does not enjoy the liking or favoritism of the student population or the general population, regardless of the educational level where we are located; therefore, the mathematics teacher must rely on various didactic strategies that allow him to capture the attention of the student body to bring them back to the classroom. That is why in this article we present a potentially significant mathematical situation, based on the study of the numerical relationships that occur on the calendar dates, specifically those that occur in the digits of the year 2020, as a support so that when the mathematics teacher teaching his class can introduce concepts or reinforce those already learned in a dynamic and motivating way.
\end{abstract}

Keywords: numerical relationships, mathematical situations, motivation, 2020, current topic.

\title{
Introducción
}

Desde la antigüedad, son muchas las culturas que se dedicaron al estudio de las diferentes particularidades que poseían los años y siglos, tanto es el caso, que aún en la actualidad escuchamos mencionar profecías provenientes de civilizaciones antiguas que establecen fechas específicas en las que, según ellos, ocurrirán ciertos acontecimientos.

Sin embargo, en la actualidad vivimos a un ritmo tan acelerado por las exigencias mundo actual y tan distraído por nuestros dispositivos móviles que no nos detenemos a observar y mucho menos a analizar aquellos hermosos detalles que a diario se nos presentan en nuestro diario vivir y en nuestro contexto en general. Es nuestro interés presentar cómo se puede aprovechar en las clases de matemática temas de actualidad, para motivar y atraer el interés del estudiantado hacia esta asignatura. En esta ocasión, mostramos como podemos lograr esto con las fechas del calendario, en particular con el año en curso (2020).

En este artículo ejemplificamos, con los dígitos del año 2020, el desarrollo de una situación matemática productiva y enriquecedora que todo docente puede utilizar en su quehacer educativo, en la que basándonos en las relaciones numéricas del año en curso se puede reforzar e introducir conceptos que no necesariamente aparecen en el currículo 
panameño, pero que, si refuerzan contenidos establecidos en él, así se aprovechan al máximo las potencialidades matemáticas que este año trae consigo.

\section{Algunas consideraciones previas}

En la última década, el sistema educativo panameño ha incorporado algunos cambios a nivel de currículo, en las diferentes asignaturas que se imparten a nivel nacional. Estos cambios conducen al docente a replantearse sus prácticas pedagógicas dentro del aula, tomando en cuenta los diferentes procesos que se dan en la misma como lo son: los cognitivos, afectivos, metodológicos y los motivacionales. Cada uno de estos íntimamente relacionados con la educación matemática y exigiendo, al docente de esta asignatura, nuevas líneas de acción dando giros en su forma tradicional de dictar sus clases que usualmente se basa en la solución de ejercicios repetitivos. Esto se logra a través de secuencias de actividades, bien estructuradas, que permitan enseñar matemática en forma efectiva mediante situaciones que despiertan el interés del estudiante hacia lo asombroso y maravilloso que le ofrece esta asignatura.

Sin embargo, en múltiples ocasiones, las clases de matemática se tornan, para algunos estudiantes, poco motivadoras, por diversas razones, ocasionando que el interés por el objeto de estudio se pierda; sin embargo, es aquí donde el docente debe apoyarse en situaciones matemáticas potencialmente significativas que atraigan la atención del alumnado. Es nuestro interés compartir con otros docentes y con la población en general situaciones que se pueden utilizar en el desarrollo de sus clases de matemática, contribuyendo tanto al desarrollo de conceptos como a su reforzamiento. Diversas investigaciones sustentan que motivar al estudiante por medio de actividades significativas en matemática produce resultados positivos en su aprendizaje.

El aprendizaje de la matemática depende, en gran medida, de los recursos que utiliza el docente dentro del aula. Según Muñiz, Alonso y Rodríguez (2014): El aprendizaje de las matemáticas puede ser una experiencia motivadora si lo basamos en actividades constructivas y lúdicas.

Tal como nos dice: Martínez O. (2007), con la incorporación de diferentes actividades lúdicas se podría contribuir a despertar la motivación de los educandos. Si, además, dichas actividades están sustentadas en objetos tales como las curiosidades matemáticas, que tienen en su esencia contenidos matemáticos que permiten explicar el porqué de lo que acontece en esas situaciones, entonces la matemática se configura 
como una actividad que debe ocupar espacios importantes en las aulas de clase, sobre todo cuando se sabe que lo lúdico se viene constituyendo en uno de los pilares fundamentales para el proceso de producción y construcción de conocimientos en el aula.

A su vez Human, Oliver y Murray (1991) citado por Mato-Vázquez (2010) tienen claro que el resultado final de las Matemáticas no depende sólo de factores intelectuales, sino que está, también, determinado por las perspectivas y experiencias de los alumnos y por la visión que ellos mismos tienen de sí como estudiantes de Matemáticas.

Un factor muy importante que debe ser tomado en cuenta y que sigue existiendo constantemente en las clases de matemática es la apatía hacia la misma. Alsina (2007) citado por Cano (2014) manifiesta "que la falta de habilidad del profesor para introducir elementos variados y motivadores acompañados de la inseguridad hacia la asignatura produce que los alumnos se desconecten de los contenidos a desarrollar".

Señala Martínez (2007), “existe la necesidad de considerar en el proceso de enseñanza-aprendizaje aspectos cognitivos como afectivos; ya que, estos conjugan aspectos intelectuales con emocionales, estos últimos considerados impulsadores clave de la actividad matemática".

Es importante acentuar que es en los descubrimientos donde el estudiante puede sentirse atraído por algo cada vez más profundo; luego, es preciso valorizarlos y, a partir de ellos, desarrollar otros más amplios, en un razonamiento de construcción. Las curiosidades matemáticas no tienen que ser desarrolladas como una solución, sino como una alternativa metodológica que permita suscitar el interés de los estudiantes por el estudio de la Matemática, Oliveira, C. y Martínez O. (2007)

El gran reto al que los matemáticos de la actualidad nos enfrentamos es lograr que nuestros alumnos conciban la matemática como una asignatura fascinante, eliminando esos prejuicios que, desde pequeños, han escuchado que trabajar matemática es aburrido, difícil, tedioso y poco motivador. Esto es posible si poco a poco vamos introduciendo en nuestras clases situaciones de aprendizaje enriquecedoras basadas en curiosidades matemáticas que despierten el interés de los estudiantes por hacer matemática, incluso, aunque no se den cuenta que están haciendo matemática. 


\section{Materiales y Métodos}

La situación matemática que sugerimos se basa en destacar las relaciones numéricas en los dígitos del año 2020. Se hace la presentación y explicación al estudiante de diferentes propiedades que pueden cumplir los números, atendiendo a los dígitos que lo forman, para que posteriormente proceda a verificar, cuál de estas propiedades se cumplen en los dígitos del año 2020.

Presentaremos definiciones de tipos de números, mismas que cumple el año 2020, con la intención de mostrar una estrategia didáctica que se puede utilizar en la gestión del aula de matemática; la cual es fácilmente adaptable a cualquier número o año calendario.

Número par: Es todo número entero que es divisible entre dos. Es decir, es un número entero que se puede escribir de la forma: $2 \mathrm{k}$, donde $\mathrm{k}$ es un entero (los números pares son los múltiplos del número 2).

Como el número 2020 puede ser escrito de la forma $2 k$, con $k=1010$, es decir:

$$
2020=2(1010)=2 k
$$

Por lo tanto, 2020 es un número par.

Número Compuesto: Es todo aquel número natural mayor que 1 que no es primo.

Como podemos apreciar esta definición hace referencia a los números primos, por lo que para verificar si este es un número compuesto se hace necesario definir también lo que es un número primo,

Número Primo: según lo definen Castelao, J. y Sánchez, L. (2000), “Es todo aquel número natural mayor que 1 que posee únicamente dos divisores, el uno y el propio número".

Por lo tanto, para saber si el 2020 es un número compuesto debemos descartar que sea un número primo. Veamos:

El número 2020 es un número par como bien se mostró en el punto anterior lo que nos indica que es divisible por 2. Esto nos lleva a que el 2020 automáticamente deja de 
tener solo 2 divisores, ya que cuenta con el 1, 2 y 2020. Si bien es cierto que posee más divisores nos basta con que tenga 3 para que deje de ser un número primo.

Con esto podemos afirmar que el 2020 es un número compuesto, ya que, no es un número primo.

Número Semiperfecto: Es todo número natural que es igual a la suma de algunos de sus divisores.

Según esta definición para que 2020 sea un número semiperfecto este debe ser el resultado de la suma de algunos de sus divisores propios. Los divisores propios de 2020 son: $1,2,4,5,10,20,101,202,404,505$ y 1010.

Si sumamos algunos de los divisores propios de 2020 tenemos que:

$2020=101+404+505+1010$

Así, 2020 es un número semiperfecto.

Número Abundante: Según Ávila, O. (2006): “un número natural que cumple que la suma de sus divisores propios es mayor que el propio número, se llama número abundante".

Si sumamos todos los divisores propios de 2020 tendríamos:

$$
1+2+4+5+10+20+101+202+404+505+1010=2264
$$

Como $2264>2020$, se tiene entonces que: la suma de los divisores propios de 2020 es mayor que el mismo 2020, lo que nos conduce a que 2020 es un número abundante.

Número Infeliz: Es todo número natural que no es un número feliz.

Esta definición nos indica que para saber si un número es infeliz primero debemos comprobar que no es un número feliz; por ende, es necesario conocer que es un número feliz.

Número Feliz: "Un número es feliz cuando la suma reiterada de los cuadrados de sus dígitos acaba siendo 1”, según Monroy, A. y Astudillo, M. (2014).

Verifiquemos si 2020 es un número feliz aplicando el procedimiento descrito: 


\section{GUACAMAYA}

Paso 1: $\quad 2^{2}+0^{2}+2^{2}+0^{2}=8$

Paso 2: $\quad 8^{2}=64$

Paso 3: $\quad 6^{2}+4^{2}=52$

Paso 4: $\quad 5^{2}+2^{2}=29$

Paso 5: $\quad 2^{2}+9^{2}=85$

Paso 6: $\quad 8^{2}+5^{2}=89$

Paso 7: $\quad 8^{2}+9^{2}=145$

Paso 8: $\quad 1^{2}+4^{2}+5^{2}=42$

Paso 9: $\quad 4^{2}+2^{2}=20$

Paso 10: $\quad 2^{2}+0^{2}=4$

Paso 11: $\quad 4^{2}=16$

Paso 12: $\quad 1^{2}+6^{2}=37$

Paso 13: $\quad 3^{2}+7^{2}=58$

Paso 14: $\quad 5^{2}+8^{2}=89$

En el paso 14, se obtuvo como resultado el número 89, que ya había sido obtenido en la suma realizada en el sexto paso, lo que nos indica que el proceso empezara a partir de ahí a repetirse entrando en un bucle, por tanto, ninguna de las sumas obtenidas ni las que se obtendrán es, ni será 1 . Esto nos lleva a afirmar que el número 2020 es un número infeliz.

Número Odioso: según la definición presentada por López, J. (2020), “es todo número cuya expresión en base 2 (binaria) contiene un número impar de unos".

Para verificar si 2020 es un número odioso primeramente debemos expresarlo en su equivalente binario, veamos: 


$$
\begin{aligned}
& 2020 \div 2=1010 \text { con residuo } 0 \\
& 1010 \div 2=505 \text { con residuo } 0 \\
& 505 \div 2=252 \text { con residuo } 1 \\
& 252 \div 2=126 \text { con residuo } 0 \\
& 126 \div 2=63 \text { con residuo } 0 \\
& 63 \div 2=31 \text { con residuo } 1 \\
& 31 \div 2=15 \text { con residuo } 1 \\
& 15 \div 2=7 \text { con residuo } 1 \\
& 7 \div 2=3 \text { con residuo } 1 \\
& 3 \div 2=1 \text { con residuo } 1
\end{aligned}
$$

Así tenemos que el equivalente binario de 2020 es:

$$
2020_{10}=11111100100_{2}
$$

Si contamos en el equivalente binario de 2020 la cantidad de 1 que aparecen obtenemos una cantidad de 7, como 7 es un número impar podemos afirmar que 2020 es un número odioso.

Número Ondulado: Es todo aquel número natural de la forma $\boldsymbol{a b a b a b}$....

Se puede observar claramente que 2020 cumple con esta definición, por lo tanto, es un número ondulado.

Número Capicúa: Según la definición dada por Cámpoli, O. (1987): “Un número capicúa es todo número natural que se lee igual de derecha a izquierda y de izquierda a derecha".

Si observamos detenidamente el número 2020, fácilmente podemos descartar que sea un número capicúa, ya que no se ajusta a la definición. Sin embargo, en este año en curso se da una fecha del calendario que si cumple las propiedades para ser un número 
capicúa; indistintamente del formato que utilice la región para indicar su fecha, siendo este día el dos de febrero de 2020.

A continuación, se muestran los diferentes formatos que se utilizar para indicar la fecha del calendario en diferentes regiones del mundo:

$$
\begin{aligned}
& \text { dd-mm-aaaa } \rightarrow \text { 02-02-2020 } \\
& \text { mm-dd-aaaa } \rightarrow \text { 02-02-2020 } \\
& \text { aaaa-mm-dd } \rightarrow \text { 2020-02-02 }
\end{aligned}
$$

Se observa que independientemente del formato utilizado, esta fecha representa un número capicúa.

La experiencia como docentes de matemática nos ha permitido vivenciar resultados positivos al utilizar este tipo de metodología didáctica, en la que se incorporan diferentes fechas del calendario que cumplen condiciones específicas ubicándolas dentro de un tipo especial de números, en la que se logra mantener al estudiante atento y motivado en todo el proceso de enseñanza aprendizaje permitiéndole aprender significativamente nuevos conceptos, así como también reforzar los ya aprendidos.

Como se mostró anteriormente en este artículo el año 2020 cumple, entre otras condiciones, la de ser un número infeliz y odioso; lo que coincide con la percepción que muchas personas tienen de él, debido a la pandemia que se está viviendo a nivel mundial a causa del covid-19.

\section{Conclusiones}

Incorporar en la clase de Matemática situaciones que ocurren en nuestro diario vivir, es una estrategia que despliega un abanico de oportunidades en el proceso de enseñanza y aprendizaje, ya que esto nos ayuda a captar la atención de los alumnos. Un alumno atento y motivado, sin lugar a duda, tendrá más oportunidades de aprendizaje que aquel estudiante que manifiesta su apatía hacia la matemática.

En este artículo, nos hemos valido del número del año en curso, 2020, para introducir una actividad productiva y enriquecedora, aprovechando las regularidades que guardan sus dígitos, lo que se puede utilizar para la introducción, el desarrollo o reforzamiento de contenidos Matemáticos. 
En la actualidad, el docente de Matemática debe valerse de múltiples estrategias y estamos seguros de que el solo hecho de iniciar una clase de Matemática diciendo: "hoy vamos a conocer números odiosos, números abundantes y números semiperfectos"; se lograría atraer la atención del estudiantado en el objeto de aprendizaje.

\section{Recomendación}

Son muchos los tipos de números que podemos encontrar de acuerdo con las condiciones que cumple cada uno, invitamos al lector a indagar en ellos y verificar dentro de que otro tipo de número se puede ubicar al 2020.

\section{Referencias Bibliográficas}

Alsina, Á., y Domingo, M. (2007). Cómo aumentar la motivación para aprender matemáticas. Suma, 56, 23-31.

Ávila, O. (2006). Acerca de los números abundantes y multiperfectos impares.

Cámpoli, O. A., \& Niell, A. M. (1987). Acerca de números palindrómicos o capicuas. Revista de educación matemática, 3(1).

Cano-Verge, N. (2014). Vivir las matemáticas: Propuesta de actividades lúdicas y significativas para el Primer Ciclo de Educación Primari (Bachelor's thesis).

Castelao, J. M. P., \& Sánchez, L. G. (2000). Sobre los números primos. Epsilon: Revista de la Sociedad Andaluza de Educación Matemática" Thales", (48), 291306.

López, J. (29 de abril de 2020). Números odiosos y malvados. El Rincón Matemático de Sherlock 221. Recuperado de https://ermds221.es/2020/04/29/numeros-odiosos-ymalvados/

Martínez, O. (2007). Matemágica: un mundo de posibilidades. Educere, 11(37), 223232.

Mato-Vázquez, D. (2010). Mejorar las actitudes hacia las matemáticas.

Monroy, A. A., \& Astudillo, M. T. G. (2014). Definir, buscar ejemplos, conjeturar... para probar si un número es feliz. Avances de investigación en educación matemática, (5), 5-24. 
cuACAMAYA

Muñiz-Rodríguez, L., Alonso P., y Rodríguez-Muñiz, L.J. (2014). El uso de juegos como recurso didáctico para la enseñanza y el aprendizaje de las matemáticas: estudios de una experiencia innovadora. Revista Iberoamericana de Educación Matemática, 19-33. 